\title{
PERSPECTIVA DE AMPLIAÇÃO DAS FUNÇÕES DA UNIDADE BÁSICA DE SAÚDE: RELATO DE EXPERIÊNCIA
}

\author{
Iranilde José Messias Mendes* \\ Ângelo Battaglion Neto** \\ Jether A. Pereira Júnior**
}

MENDES, I.J.M.; NETO, A.B.; JÚNIOR PEREIRA, J. A. Perspectiva de ampliação das funções da unidade básica de saúde: relato de experiência. Rev.latinoam.enfermagem, Ribeirão Preto, v. 4, n. 1, p. 119-29, janeiro 1996.

Os autores relatam uma experiência de atenção primária à saúde à partir de uma abordagem holística de atenção ao homem, enfatizando os aspectos de promoção da saúde dentro do modelo epidemiológico "O Campo de Força e Paradigma de Bem Estar".

Esta experiência engloba atividades de exercícios físicos, lazer, excursões e curso sobre a saúde individual e ambiental para 255 adultos com idades de 35 a 80 anos em uma praça pública nas imediações de uma Unidade Básica de Saúde (UBS).

Alertam sobre a necessidade dos políticos e serviços de saúde redirecionarem suas atividades numa concepção abrangente de saúde.

UNITERMOS: promoção da saúde, auto cuidado, habilidade de vida, habilidades sociais

\footnotetext{
* Professor Doutor do Departamento de Enfermagem Materno-Infantil e Saúde Publica da Escola de Enfermagem de Ribeirão Preto da Universidade de São Paulo

**Professor de Educação Física do Centro de Educação Física e Recreação de Ribeirão Preto da Universidade de São Paulo
} 


\section{INTRODUÇÃO}

A redução da taxa de mortalidade e, principalmente o declínio da fertilidade tiveram como conseqüência uma alteração na composição etária da população mundial. As Nações Unidas calculam que no ano dois mil e vinte e cinco só um quarto das pessoas em todo planeta terá menos de quatorze anos de idade e que, do total da população com sessenta anos e mais, setenta por cento viverá em um mundo em desenvolvimento ${ }^{12}$.

O envelhecimento da população tem tido como característica o desenvolvimento de certas doenças não transmissíveis, cujas causas têm sido atribuídas ao estilo de vida. Entre elas, destacam-se enfarte do miocárdio, trombose, hipertensão arterial, câncer, doenças pulmonares, biabetes, osteoporose e artrite. Essas doenças são responsáveis por setenta a oitenta por cento das mortes no mundo desenvolvido e quarenta a cinqüenta por cento no mundo em desenvolvimento ${ }^{1,3.6 .7 .9,11,12,16,17,18,20,22}$.

Acredita-se que a prevenção dessas doenças só será possível dirigindo-se a atenção aos fatores de risco relacionados com o seu desenvolvimento. Fatores tais como a poluição do ar e a má distribuição de renda apresentam características que dificultam o controle a nível individual e, portanto necessitam de ação governamental e de organização da sociedade. Muitas outras, porém, estão diretamente relacionadas com escolhas pessoais. Reconhece-se até mesmo que o indivíduo poderá alcançar uma vida longa e saudável e ter inclusive uma morte fisiológica desde que a partir da infância adote um modo de vida sensato ${ }^{12,16}$.

A OMS (Organização Mundial da Saúde), vem chamando a atenção das Nações para a necessidade da prevenção das doenças produzidas por estilo de vida. São doenças com causas comuns e que devem ser combatidas em conjunto através de uma estratégia e prevenção comum ${ }^{12}$.

Já se dispõe de estudos epidemiológicos suficientes dos fatores de risco. $\mathrm{BLUM}^{3}$, classifica esses riscos em três grupos: riscos da atividade de lazer (falta de exercício físico e recreação); risco de padrão de consumo (excesso de alimentação, ingestão de colesterol, consumo de bebida alcoólica, fumo, dependência de drogas, entre outras) e riscos ocupacionais (estresse no trabalho, dirigir sem prudência, entre outros).

Os sistemas de saúde mundial atualmente estão sendo questionados a respeito da sua prática, sustentada predominantemente na concepção negativa de saúde $6.9,14.15,17.21$.

Vimos testando propostas numa concepção positiva de saúde com alunos de graduação e pessoal de serviço e comunidade. Alguns resultados estão sendo divulgados ${ }^{15}$.

A preocupação de uma maior aderência da questão do ensino com prática a assistência de forma a melhor adequar a filosofia do ensino empreendido no Curso de Graduação em Enfermagem na disciplina Saúde do Adulto, levaram-nos a elaborar em 1993 um projeto de trabalho embasados nos pressupostos do modelo 
epidemiológico de BLUM ${ }^{3}$, com o objetivo de estimular a Unidade Básica de Saúde a ampliar o seu papel na perspectiva de torná-la um local onde seus clientes e trabalhadores possam se transformar, visando o auto-cuidado e a responsabilidade individual pela própria saúde, e também de proporcionar um meio que fosse estimulante à participação de grupos comunitários, levou-nos a buscar uma fundamentação teórica para a implementação desta experiência prática.

\section{REFERENCIAL TEÓRICO}

A concepção do estudo é o que está expresso na Carta de Otawa ${ }^{24}$ e a conformação de seu conteúdo obedece aos preceitos do modelo epidemiológico proposto por BLUM ${ }^{3}$.

O modelo epidemiológico tradicional de doenças com três componentes: agente, hospedeiro e ambiente. Foi desenvolvido quando as doenças infecciosas eram a preocupação principal dos epidemiologistas. Com a mudança nos padrões de doença e no enfoque dos estudos epidemiológicos, novos modelos foram desenvolvidos, ampliando o modelo tradicional, reduzindo a importância do agente e ampliando a dos fatores ambientais e o hospedeiro $3,4,6,9,14$.

Para responder a estas mudanças, surgiram alguns modelos epidemiológicos. Em um mil novecentos e setenta e três, LA FRAMBOIASE \& LALONDE $^{6}$ apresentaram um modelo de saúde com objetivo de reduzir doenças; denominado "conceito de campo de saúde", o qual sustenta que a saúde é determinada por uma variedade de fatores agrupados em quatro divisões principais: estilo de vida, ambiente, organização dos cuidados com a saúde e biologia humana. Em um mil novecentos e setenta e quatro, BLUM3 amplia a concepção do modelo, com o objetivo de relacionar saúde e bem estar denominando-o "ambiente de saúde", posteriormente reintitulado "O Campo de Força e os Paradigmas do Bem Estar de Saúde".

$\mathrm{BLUM}^{3}$ faz uma abordagem mais abrangente do paciente que apresenta problemas de saúde, sugerindo que todos os serviços de apoio ao Ser Humano devem ser integrados desde os serviços de atenção primária, porta de entrada para aqueles que apresentam agravos ou problemas de qualquer espécie. As manifestações em uma esfera podem ser causadas por sobrecargas em outras. Os problemas de doença, considerados por $\mathrm{BLUM}^{3}$ como problemas mais simples, devem ser resolvidos a nível curativo, enquanto que as causas subjacentes àquelas fases críticas devem ser trabalhadas em níveis mais complexos como a família, ocupação, emprego, moradia, resistência a infecções ou qualquer que seja a causa. Quando indicada, a busca será dirigida aos fatores de risco, predisponentes ou agravantes, presentes nas chamadas causas primordiais. Com isso, deixa claro que os prestadores de serviço precisam estender seus serviços à comunidade, num esforço de atender não apenas os seus pacientes, mas também a todos aqueles que estão sob riscos 
ambientais, estilo de vida ou hereditariedade. Os serviços de saúde devem ter um compromisso com a saúde da comunidade como um todo e com seus membros individualmente, envolvendo-os nos programas antes mesmo que eles adoeçam, ou seja, preparando-os para reconhecer situações agravantes da doença e sendo capazes de auto-cuidar-se, motivados a assumir maior responsabilidade pela própria saúde.

O conceito de Campo de Saúde é mais abrangente e se adapta melhor à visão "múltipla causa/múltiplo efeito" da saúde e doença e a ampliação dos conceitos de risco. Apresenta implicações diretas de mudança social, pois de acordo com este conceito, só há doença se houver desequilíbrio em alguma das etapas do campo de força. Este modelo proporciona uma abordagem ma para o desenvolvimento de uma política de saúde quando comparada ao modelo de saúde pública tradicional.

Assim, a análise destes campos de saúde resultaria em doze aspectos mensuráveis do estado de saúde somática, psíquica e social do homem: expectativa de vida, doença, desconforto, incapacidade, participação nos cuidados com a saúde, comportamentos saudáveis, comportamento ecológico, comportamento social, interpessoal, resistência às doenças (reserva), satisfação interna e externa.

$\mathrm{BLUM}^{3}$, dispõe o campo de força em forma de círculo, situando o bem estar no centro. Sendo assim, todos os elementos, do campo de força agem em conjunto, numa relação de equilíbrio, e o bem estar é avaliado por elementos mensuráveis que estão ligados à saúde somática, social e psíquica do homem. Se houver desequilíbrio em alguma dimensão, esteja ela situada em qualquer ponto da escala hierárquica de importância do campo de força, será refletida no bem estar e manifestará seu desequilíbrio através dos doze elementos mensuráveis ligados diretamente ao homem.

Este modelo é também visto como uma teoria de mudanças sociais, pois seu pressuposto para a saúde envolve uma sociedade organizada e responsável pelo auto-cuidado.

A Carta de Otawa ${ }^{24}$, reflete o espírito do modelo de $\mathrm{BLUM}^{3}$ quando defende promoção de saúde como "um processo que confere às populações os meios de assegurarem um maior controle sobre sua própria saúde e de melhorá-la". Estas condições envolvem paz, abrigo, alimento e uma renda, conseguidos através de uma política social organizada.

\section{METODOLOGIA}

Para início do trabalho mais sistematizado de atividade física e recreacional, buscamos articulação com o CEFER (Centro de Educação Física e Recreação de Ribeirão Preto da Universidade de São Paulo), para uma possível colaboração dos profissionais desta área, o que foi prontamente atendida e oficializada através do 
intercâmbio Escola de Enfermagem e Prefeitura do Campus.

Com a preocupação de motivar e garantir a participação dos profissionais da Unidade Básica de Saúde (UBS) na discussão e encaminhamento do projeto, foram proporcionado dois encontros na própria unidade com um professor de Educação Física, que discutiu os conhecimentos da área na melhoria da qualidade u de vida e no controle das doenças crônicas.

Para início das atividades, divulgamos a proposta do projeto a todos os trabalhadores da UBS, onde trabalhamos com estagiários há três anos e solicitamos aos médicos que encaminhassem seus clientes à praça para participarem das atividades propostas; o que foi prontamente atendido.

A equipe responsável pelo desenvolvimento do trabalho foi constituída de duas enfermeiras e dois professores de Educação Física, autores deste relato.

\section{DESENVOLVIMENTO DO TRABALHO}

A partir de novembro de 1993, começamos as atividades práticas. Naquela ocasião compareceram doze clientes com diagnóstico médico de hipertensão e/ou diabetes. Dado a propósito do programa de não só trabalhar com pessoas portadoras de doenças crônicas, mas também com pessoas supostamente sadias foi-se ampliando o número de participantes ${ }^{10}$. A forma como se dava o acesso deles às atividades foi muito simples e livre de praticamente toda burocracia. Ao verem o grupo trabalhar, as pessoas se dirigiam a qualquer um dos responsáveis pelo programa, indagavam sobre o que deveriam fazer para participar do mesmo. Diante da resposta de que, não ha vendo restrição médica, era necessário somente sua identificação e endereço, o que se fazia no mesmo local, a procura foi aumentando de tal maneira que até no mês de março de 1994 havia 80 participantes e, atualmente, mês de outubro o grupo é constituído de 225 adultos, com idade entre 35 e 80 anos, sendo 201 mulheres e 24 homens.

O Programa, que recebeu a denominação de PIC (Programa de Integração Comunitária) por um dos participantes, é desenvolvido três vezes por semana, no horário das sete às oito horas da manhã, numa praça pública de um bairro antigo da cidade, próximo a UBS. As atividades compreendem 30 a 40 minutos de caminhada, onde todos participam. Para essa atividade é respeitado o limite de cada um, à medida que as pessoas vão melhorando em condicionamento físico, elas vão aumentando o nível e grau dessa atividade. Para isto, utiliza-se o método descrito por COOPER ${ }^{8}$, que utiliza como parâmetro tempo e percurso, seja de corrida ou caminhada. Decorrido esse tempo, as pessoas são subdivididas em três grupos, quando durante mais 30 minutos, um grupo fica com exercício físico, um outro 
desenvolve atividades de recreação e o terceiro grupo, freqüenta aulas de educação em saúde. Tais aulas fazem parte de um programa teórico-prático, cujo conteúdo tem como objetivo estimular a clientela para:

a) refletir sobre sua relação com o meio ambiente;

b) discutir seu papel de cidadão no contexto da sociedade brasileira;

c) identificar fatores e grupos de risco populacionais;

d) adquirir conhecimentos básicos, atitudes e habilidades com vistas à mudança de comportamento.

Para manter os grupos sempre motivados é feito o remanejamento entre eles de tal forma que nas três vezes por semana os três grupos participam das três atividades. São organizadas ainda pequenas excursões a clubes e áreas de lazer da cidade, atividades essas que despertam um interesse muito grande de todos os participantes.

\section{CONSIDERAÇÕES FINAIS}

Observa-se na literatura consultada, um interesse pelo estudo da saúde por si só e uma ênfase especial tem sido dada às abordagens e atividades para manter e ampliar os níveis de saúde existentes.

Essa vivência tem nos mostrado que é possível dirigir essas ações enfatizando os aspectos da promoção da saúde e, desse modo, contribuir para o redirecionamento das ações em nível de Unidade Básica de Saúde.

Depoimentos de participantes como "Depois que comecei vir aqui os problemas sumiram", ou "Eu diminui a quantidade de remédios que tomava e tenho mais agilidade no corpo" ou ainda "o médico sempre me mandou caminhar e, eu até tentava, mas não tinha estímulo, tinha até vergonha" ou ainda: "estou ótima, já perdi cinco quilos e não dependo mais de remédio". "Estou aprendendo muitas coisas. Se tivesse essa oportunidade antes, teria sido bem mais feliz". "Depois que comecei vir aqui os problemas sumiram e eu já não mais me contento com os dias do PIC, ando todas as manhãs. Agora eu sou muito mais feliz. O PIC serviu para melhorar a convivência da comunidade. Muitas pessoas só se conheciam de vista, aqui elas conversam e se tomam mais amigas".

Essas e outras manifestações ouvidas diariamente pelos organizadores do programa têm levado-os a questionarem as políticas e as ações dos serviços de saúde que, ao atuarem somente no interior das UBS e tratarem quase que exclusivamente dos aspectos biológicos, parece-nos que estão longe de se aproximarem das necessidades da população.

Esses depoimentos nos levam a concordar com QUEIROZ ${ }^{19}$ quando diz 
que a manutenção do modelo curativo centrado na biologia e no hospital não é adequada para compreender, interpretar, explicar e resolver amplos aspectos relacionados à saúde e à doença. $E$, neste particular, preocupa-nos a dificuldade dos profissionais da saúde em realizar um trabalho articulado com outros profissionais, num intercâmbio de saberes, uma vez que de um modo geral, eles sentem-se inteiramente responsáveis por uma saúde, que deveria ser vista como a possibilidade de desenvolver habilidade de vida, auto-conceito e habilidades sociais e, também se preocupar com a intervenção através de amplos recursos políticos, legislativos, fiscais e administrativos (STACHITCHENKO) ${ }^{21}$. Assim, a promoção de saúde, aqui entendida, abrange alguns aspectos da saúde pública atual no que diz respeito ao atendimento especializado em suas Unidades Básicas, e referentes aos cuidado com estilo de vida e o meio ambiente, abrindo espaço para uma atuação multidisciplinar que, pode atuar em outros segmentos da sociedade que dizem respeito a cultura do grupo social, alimentação, habitação, renda, acesso e posse de terra, crenças, valores e vida espiritual.

Este trabalho tem nos levado a sentir que, a atuação do enfermeiro junto à promoção de saúde requer habilidades no campo da sociologia, antropologia, comunicação, epidemiologia, entre outros, e que pode atuar como mediador (negociador) junto a equipe multiprofissional das áreas sociais e biológicas e a população.

Um outro aspecto positivo e fácil de constatar foi a queda na freqüência daqueles clientes à Unidade Básica a procura de consulta médica, após a implantação do programa.

Segundo informações do gerente da Unidade Básica a distribuição de analgésicos e anti-hipertensivos diminuiu em trinta por cento. $\mathrm{E}$, para os trabalhadores da Unidade Básica, começa-se a perceber uma mudança no comportamento dos clientes.

Tais constatações nos levaram a crer na necessidade de implementar esse programa em outras unidades e desenvolver concomitantemente investigações, tanto procurando avaliar o impacto do programa, tendo como parâmetro melhor utilização da UBS pelos usuários; aptidão física e saúde da clientela, mudanças de comportamento a nível individual (mudança no hábito alimentar, adoção da prática do exercício físico, entre outras) e social (participação em grupos comunitários, interferência no meio ambiente, entre outras); devendo-se buscar a base conceitual para tais estudos na Educação Física, Antropologia, Epidemiologia, Sociologia e outras ciências ditas sociais e biológicas.

Dentre essas considerações, surgiu a questão sobre de que maneira o enfermeiro pode contribuir para a saúde das pessoas. Dentro deste modelo, epidemiológico o "Campo de Força e o Paradigma de Bem Estar" e da experiência vivenciada neste campo de trabalho, podemos antever que a ênfase deverá ser dada a um processo educativo, no qual o enfermeiro e outros profissionais da equipe compartilhem saberes, sem haver hegemonia de um ou outro profissional. Desta 
maneira poderão agir sobre o significado das propostas de saúde modificando-as ou confirmando-as e utilizando as forças positivas disponíveis para enfrentar a gama de problemas que envolvem as possíveis causas da doença. Todo esse processo, certamente não ocorrerá sem haver um redirecionamento das ações de saúde e dos próprios profissionais no sentido de repensar suas ações. Este requer um constante enfrentamento, principalmente referente a manutenção da adesão a certas restrições ou mudanças do estilo de vida, e portanto, este processo contínuo poderá levar também a um contínuo convívio entre enfermeiro e a equipe, de modo a possibilitar uma interação efetiva de grupos na busca de um trabalho comum, sem arrogância intelectual, pois nenhum grupo tem domínio do conhecimento ou inovações, o processo se dá pela interação de ações e pensamentol ${ }^{3}$.

Cabe ainda assinalar a importância que tem o vínculo pessoal mantido entre profissionais e clientela. No presente trabalho, os autores juntamente com os participantes, se envolvem num relacionamento interpesssoal, de tal modo que há momentos que é difícil distinguir o profissional da clientela.

Tais situações nos remetem a Maclen \& Eakin ${ }^{13}$, quando assinalam que as atividades de promoção de saúde se constituem em experiência tão envolvente, carismática e, às vezes, com características de movimento religioso. Por isso, devemos estar atentos para assegurar a honestidade e o rigor intelectual, para não se deixar convencer pelo carisma ou poder que ela envolve, mas pela mudança social que ela cria. Esta capacidade de mudança do ser humano e criação de novas possibilidades individuais e no contexto social se constitui em uma realidade experienciada pelos autores no contato e interação com os clientes, onde homens e mulheres que partilham de diferentes vivências culturais e posições sociais, procuram mudar conceitos criados e mantidos pelo próprio homem com o objetivo de viver melhor.

Um outro aspecto que deve ser considerado é a possibilidade de enfrentamento de conflitos. Trabalho desta natureza dá oportunidade à população de um maior desenvolvimento de participação comunitária ajudando-a, desse modo, a se organizar a nível familiar e comunitário, possibilitando aquisição de maior controle sobre sua vida e saúde. Por outro lado, nossas instituições estão norteadas por uma visão de mundo pragmática, onde a medida de tudo é o poder político e econômico, impedindo um trabalho conjunto que possa levar a uma sociedade sensata, mais justa e mais humana. E, neste particular, chamamos a atenção para o papel do profissional da área da saúde que, como as instituições, necessitam refletir sua concepção de mundo. Concordamos com WERNER ${ }^{23}$, quando diz que: "a visão daqueles que dirigem os programas de saúde comunitária quer seja uma visão compartilhada por muitos ou a visão eletista de alguns poucos, quer seja uma visão autoritária ou humanitária, terá maior impacto sobre o programa do que as metas e os objetivos estabelecidos".

Esta experiência tem nos mostrado que é possível redirecionar nossas ações a níveis primários de saúde, bem como estudar e utilizar metodologias 
científicas que nos permita avaliar situações em que se observa melhora na qualidade de vida, satisfações humanas, comportamento social, entre outras mudanças que se constituem objetivos da promoção de saúde.

A divulgação de uma avaliação sistematizada, baseada em um rigor científico se constituirá em objeto de publicação posterior, pois acreditamos na relevância do projeto de investigação dirigida à promoção da saúde, a fim de que se possa aprofundar o conhecimento da temática. Os resultados contribuirão, sem dúvida, para redirecionar políticas e serviços de saúde.

\section{PERSPECTIVES OF EXPANSION OF BASIC HEALTH UNITS FUNCTIONS: A REPORT OF AN EXPERIENCE}

The authors report an experience of primary health care emphasizing aspects of Health Promotion within the Epidemiologic Model: "The field of force and the Paradigm of well-being".

This experience combines physical exercises, leisure, excursions and a course about individual and environmental health to 255 adults (35 to 80 years old) in a square near the Basic Health Unit.

The authors mention the necessity of a new arrangement of health services activities comprehending a wide conception of health.

UNITERMS: health promotion, self-care, life ability, social ability

\section{PERSPECTIVAS DE AMPLIACIÓN DE LAS FUNCIONES DE LA UNIDAD BÁSICA DE SALUD: RELATO DE EXPERIENCIA}

Los autores relatan una experiencia de atención primaria en Salud, a partir de un abordaje holístico de atención al hombre, enfatizando los aspectos de Promoción de a Salud dentro del Modelo Epidemiológico: "El Campo de fuerza y el paradigma del bienestar".

Esta experiencia engloba actividades de ejercicios físicos, pasatiempo, excursiones y curso acerca de la salud individual y ambiental para 255 adultos con edad de 35 a 80 anos, realizados en una plaza de las proximidades de la Unidad Básica de Salud (UBS).

Alertan sobre la necesidad de una orientación de las actividades de los servicios de salud en una concepción amplia de salud.

UNITERMOS: promoción de la salud, auto cuidado, habilidad de vida, habilidades sociales 


\section{REFERÊNCIAS BIBLIOGRÁFICAS}

01. BANCO MUNDIAL. Novo desafio à saúde do adulto. Washington: Banco Mundial, 1991. p. 134 (Série de Estudos do Banco Mundial Sobre Países).

02. BARBANTI, V. J. Aptidão física: um convite à saúde. São Paulo: Manole, 1990.

03. BLUM, H. L. Planning for health, development and application of social change theory .Human Science. 2. ed. New York: Human Sciences Press, 1981.

04. BLUE CROSS ASSOCIATION UNITED STATE AMERICAN. Blue print for health help yourself. USA, v. 28, n. 1, 1978.

05. BRASIL. República Federativa do Brasil. Constituição (1988), Artigo 198. Brasília, Senado Federal, Centro Gráfico, 1988.

06. CAPRA, F. O ponto de mutação: a ciência, a sociedade e a cultura emergente. São Paulo: Cultrix, 1993.

07, COOPER, K.H. Controlando o colesterol. Trad. Anilde Wernwck. Rio de Janeiro: Nórdica, 1988. p. 345.

08. .O programa aeróbico para o bem estar total. Trad. Luís Horácio de Matta. Rio de Janeiro: Nórdica, 1982.

09. DEVER, G. E. A. A epidemiologia na administração dos serviços de saúde. São Paulo: Pioneira, 1988.

10. Exercícios físicos e lazer reduzem consumos de remédios. O Estado de São Paulo, São Paulo, 07 de julho de 1994. C-10. Cidades.

11. KALACHE, A. et al. O envelhecimento da população mundial. Um desafio novo. Rev.Saúde Pública, São Paulo, v. 21, n. 3, p. 200-10, 1987.

12. KHALTAEV, N. Intersaúde luta contra as doenças por estilos de vida. Saúde Mundo, Ginebra, p. 18-20, mai/jun. 1991.

13. MACLEAN, H. M.; EAKIN, J. M. Health promotion research methods: expanding the repertorre. Can. J .Public Health, Ottawa, v. 83, p. 84-5, mar/apr 1992. Supplement 1.

14. MENDES, E. V. A crônica de uma morte anunciada ou nascimento, paixão, morte e ressurreição do INAMPS. Brasília: OPAS/OMS, 1993. (mimeografado).

15. MENDES, I.J.M. et al. Um programa sobre promoção da saúde do adulto para trabalhadores em saúde. Rev.latino-arn.enfermagern, Ribeirão Preto, v. 2, n. 1, p. 95-108,1994.

16. NAKAJIMA, H. A saúde das nações está a alterar-se. Saúde Mundo, Ginebra, p. 18-20, mai/jun, 1991.

17. ORGANIZACIÓN PANAMERICANA DE LA SALUD/OMS. El papel de enfermería en el cuidado del adulto con énfasis en la promoción de la salud. Washington: OPAS/OMS, 1992. p.34. 
18. POSNER, M.B. et al. Comer com inteligência para viver mais. A Saúde do Mundo. mai/jun, 1991. p. 22-4.

19. QUEIROZ, M. S.; VIANA, A. L. Padrão de política estatal em saúde e o sistema de assistência médica no Brasil. Rev.Saúde Pública, São Paulo, v. 26, n. 2, p. 132-40, 1992.

20. RAMOS, L.R. et al. Envelhecimento populacional: uma realidade brasileira. Rev.Saúde Pública, São Paulo, v. 21, n. 3, p. 211-24, 1987.

21. STACHITCHENKO, S.; JENICEK, M. Conceptual differences between prevention and health promotion: research implications for community health programs. Can. J. Public Health, Ottawa, v. 81, p. 53-9, jan/fev, 1990.

22. VERAS, R.P. et al. Crescimento da população idosa no Brasil: transformações e conseqüências na sociedade. Rev.Saúde Pública, São Paulo, v. 21, n. 3, p. 225-33, 1987.

23. WENER, O. Além de objetivos e metas: a visão. TAPS saúde da comunidade: um desafio. São Paulo: Ed. Paulinas, 1984, p. 5. (Coleções Cadernos Contact).

24. WORLD HEALTH ASSOCIATION. Ottawa charter for health promotion. In: AN INTERNATIONAL CONFERENCE ON HEALTH PROMOTION. Canadá, 17-21, October, 1986. Canadá: WHO/Health and Welfare, Canadá Public Health Assoc., 1986. 